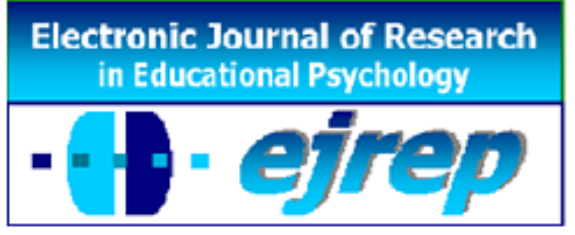

\title{
Mejora de la satisfacción corporal en chicas y chicos preadolescentes: \\ Efectos a corto plazo de un programa escolar
}

\author{
Arnold Hinz
}

Instituto de Ciencias de la Educación

Universidad de la Educación de Ludwigsburg

\begin{abstract}
Alemania
Correspondencia: Arnold Hinz. Institute of Educational Sciences, Ludwigsburg University of Education, Reuteallee 46, D-71634 Ludwigsburg. Alemania. E-mail: hinz@ph-ludwigsburg.de

(C) Education \& Psychology I+D+i e Ilustre Colegio Oficial de la Psicología de Andalucía Oriental (Spain)
\end{abstract}




\section{Resumen}

Introducción. El objetivo de esta investigación ha sido evaluar el programa de prevención escolar Mi cuerpo y yo. El programa fue desarrollado para que los niños y niñas fueran mejor preparados de cara a los cambios inevitables de la pubertad.

Método. Para este estudio se empleó un modelo de grupo experimental y grupo control en colegios de Alemania y Francia; hubo 25 clases experimentales y 17 clases de control (484 niños y 485 niñas; edad media de 10,5 años); se midieron los cambios entre pretest y postest.

Resultados: En comparación con las clases de control, las clases experimentales muestran un incremento significativo de conocimiento (tamaño de efecto $=0.89$ ), una reducción en la insatisfacción con su cuerpo $(\mathrm{TE}=0.12)$, y en la preocupación por la forma corporal $(\mathrm{TE}=0.11)$, así como un alejamiento del ideal de un cuerpo delgado $(\mathrm{SE}=0.13)$.

Discusión y Conclusiones. Los resultados de esta investigación sugieren que el Programa $M i$ cuerpo y yo es eficaz para la reducción de la insatisfacción corporal en niños y niñas de cuarto y quinto curso de Educación Primaria.

Palabras Clave: Prevención, educación primaria, insatisfacción corporal, factores de riesgo. 


\begin{abstract}
Introduction. The aim of this study was to evaluate the school-based "My Body and I" program which was designed to help girls and boys to cope better with inevitable pubertal body changes.
\end{abstract}

Method. Using a pre-post treatment-control design with 25 treatment and 17 control classes from schools in Germany and France (484 boys, 485 girls; mean age: 10.5 years), changes were measured between a pretest and a posttest.

Results. Compared with the control condition, students in the treatment condition exhibited a significant increase in knowledge (effect size $=0.89$ ), a decrease in body dissatisfaction (ES = $0.12)$, body shape concerns $(\mathrm{ES}=0.11)$, and a shift away from the thin ideal $(\mathrm{ES}=0.13)$.

Discussion and Conclusion. The findings suggest that "My Body and I" is an effective primary prevention program reducing the risk for body dissatisfaction among 4th and 5th graders.

Keywords: prevention, elementary school, body dissatisfaction, body image, risk factors. 


\section{Introducción}

La satisfacción corporal se puede definir como el grado de coincidencia entre la percepción del cuerpo real propio, y el cuerpo ideal que desea. Una mayor discrepancia indicaría poca satisfacción corporal. Varios estudios pudieron detectar un impacto de la satisfacción corporal sobre el autoestima, la auto-aceptación, y los comportamientos saludables (Mond, van den Berg, Boutelle, Hannan, y Neumark-Sztainer, 2011; Walker, 2007).

Los cambios físicos de la adolescencia son importantes y no ocurren simultáneamente. Por lo tanto, las niñas y los niños suelen pasar por dificultades a la hora de adaptarse a nuevas formas físicas, que a veces resultan extrañas y poco elegantes. Debido a las influencias socioculturales y los cambios físicos en el inicio de la pubertad, los niños se empiezan a preocupar por el tamaño corporal y la musculatura, y las niñas con su aspecto físico y su peso (Markey y Markey, 2011). Durante millones de años, la capacidad de almacenar reservas de grasa ha sido vital para la supervivencia de las especies. Las mujeres forman depósitos de grasa subcutánea en las caderas, muslos, nalgas, pechos y zona púbica. Estos depósitos de grasa facilitan la buena salud durante el embarazo, y compensan la mayor demanda de energía durante la lactancia. La acumulación de grasa se acelera en ambos sexos, pero es más rápida en las niñas que en los niños. En el inicio de la adolescencia, aproximadamente la mitad de las diferencias en aspecto físico entre los dos sexos se debe a la diferencia en grasa corporal (Bird, 2006; Malina y Bouchard, 1991; Steinberg, 2010; Susman y Rogol, 2004). Resulta sorprendente que, según un estudio realizado por Smolak, Levine, y Schermer (1998a), sólo el 17\% de los alumnos de quinto de Primaria saben que la pubertad da lugar a cambios de grasa corporal.

Investigaciones previas han demostrado que la exposición al ideal mediático de la delgadez (p.ej. en la televisión, revistas, cine, vallas publicitarias, Internet), constituye un factor de riesgo de la preocupación sobre la imagen corporal y los trastornos alimenticios (LópezGuimerà, Levine, Sánchez-Carracedo, y Fauquet, 2010). Los ideales mediáticos de la atracción física, tanto masculinos como femeninos, favorecen la delgadez y rechazan el sobrepeso. Las chicas deben ser delgadas, y los chicos deben ser dotados de músculos bien definidos en los brazos, el pecho y los hombros (Lunde, 2009). Muchos trabajos experimentales manifestaron que la satisfacción corporal de los participantes disminuyó después de ver modelos delgados idealizados (Sepúlveda y Calado, 2012). Por tanto, la deconstrucción de las imágenes mediáticas es una herramienta importante para prevenir la insatisfacción corporal. La insatis- 
facción corporal por sí sola puede ser causa de angustia, y ha sido identificado como el principal factor de riesgo de los trastornos alimenticios (Stice, 2002). Frente al riesgo de morbilidad y de muerte prematura debido a los trastornos alimenticios (Bennaroch, Pérez, \& Perales, 2011; Steinhausen, 2002), y a la inversión de energía, tiempo y dinero en busca de un ideal poco realista de delgadez, la prevención de la insatisfacción corporal es un enfoque tanto económico como humano.

Stice y Shaw (2004) encontraron que los programas de prevención de trastornos alimenticios eran más eficaces cuando se centraban en participantes de más edad (hasta los 15 años). Existen muchos aprendizajes valiosos que surgieron de este meta-análisis ampliamente citado. Sin embargo, puede ser poco ético retrasar la intervención hasta que los estudiantes ya hayan desarrollado la insatisfacción corporal (Dohnt y Tiggemann, 2008). Hasta ahora, los esfuerzos preventivos se han centrado en la etapa adolescente, y poca atención se ha dedicado a la etapa prepúber. La mayoría de los estudios de prevención para niñas y niños preadolescentes eran evaluaciones sin control (véase las revisiones realizadas por Levine y Smolak, 2001, 2007; Piran, 2005; Holt y Ricciardelli, 2008; O’Dea, 2005). Encontramos sólo cuatro estudios bien diseñados sobre la prevención desde la escuela (con un diseño pretest-postest, grupo de control y mediciones fiables) para niños de $4^{\circ}$ y $5^{\circ}$ de primaria. Smolak, Levine, y Schermer (1998a, 1998b) desarrollaron el programa escolar para primaria "Eating smart, eating for me" [«Comer con cabeza, comer para mí»] (diez lecciones), y observaron un incremento de conocimiento, y una disminución de actitudes negativas hacia las personas con sobrepeso, pero no hubo cambios en la estima corporal. Otro programa desde la escuela primaria (11 lecciones), diseñado para mejorar la satisfacción corporal, se llama "Healthy Body Image: Teaching Kids to Eat and Love Their Bodies, Too!” [«Imagen corporal saludable: enseñanza para que los chicos coman bien, y que quieran también a sus cuerpos»] (Kater, Rohwer, y Levine, 2000). Empleando un diseño de pre y post tratamiento con control, Kater, Rohwer, y Londre (2002) encontraron cambios significativos y positivos para los varones del grupo experimental en todas las medidas. Sin embargo, para las niñas sólo observaron efectos en conocimiento y alfabetización mediática, pero no en imagen corporal, en prejuicio sobre tamaño del cuerpo, en comportamiento de estilo de vida, ni en auto-imagen. Stock et al. (2007) desarrollaron y evaluaron un programa de prevención desde la escuela primaria que se llama "Healthy Buddies" [«Amiguetes saludables»], basado en enseñanza entre iguales. No encontraron efectos significativos de la intervención en percepción de la imagen corporal, en auto-competencia, o en actitudes alimenticias. Más recientemente, Bird, Halliwell, Diedrichs, 
y Harcourt (2013) evaluaron una versión adaptada del programa "Happy Being Me" [«Contento de ser yo mismo»] (Richardson \& Paxton, 2010), con niños de 10-11 años en la escuela primaria. Para niñas, encontraron mejoras significativas en satisfacción corporal, comparaciones de aspecto, y comportamientos alimenticios, para niños, no encontraron impacto significativo en la satisfacción corporal. En resumen, sigue existiendo una necesidad de programas de prevención desde la escuela, dirigidos a niñas y niños de $4^{\circ}$ y $5^{\circ}$ de primaria, eficaces para mejorar la satisfacción corporal de ambos sexos.

El propósito de este trabajo ha sido el desarrollar un programa coeducativo de prevención desde la escuela, eficaz para mejorar la satisfacción corporal en preadolescentes. Los programas escolares para mejorar la satisfacción corporal deben ser coeducativos: primero, para que haya oportunidades de aprendizaje que provengan de la presencia de ambos sexos; segundo, para ayudar a reducir las burlas basadas en peso o la forma corporal (los niños y niñas de la clase son parte del contexto de iguales, y tienen un papel importante en perpetuar el ideal de la imagen corporal); y tercero, con vistas a una más fácil implementación en las escuelas coeducativas (las escuelas primarias de Alemania suelen ser coeducativas). El programa de prevención Mi cuerpo y yo (Hinz \& Denner, 2007) tiene duración de cinco horas, y fue diseñado para niños de $4^{\circ}$ de primaria en clases coeducativas. Fue evaluado con un diseño experimental pre-post, con control $(\mathrm{N}=398)$. Un efecto significativo de la intervención sólo se encontró para la variable dependiente «Conocimiento del desarrollo puberal». Para este trabajo, el programa fue ampliado con una unidad sobre alfabetización mediática (dirigida a alumnos de $4^{\circ}$ y $5^{\circ}$ de primaria), y fue evaluado con otra muestra más grande. La alfabetización mediática debe ser un objetivo de cualquier programa de prevención de la insatisfacción corporal, debido al efecto adverso de la exposición a la imagen corporal mediático, la interiorización del ideal delgado, y la preocupación por el peso (Becker, 2004; Birkeland et al., 2005; Cohen, 2006). El fundamento teórico del programa preventivo Mi cuerpo y yo es la teoría del afrontamiento proactivo (Schwarzer \& Taubert, 2002). Aunque el afrontamiento ha sido concebido principalmente como una respuesta de reacción, en la actualidad ha llegado a ser considerado algo proactivo y orientado a futuro (Greenglass, 2002). Se considera que los problemas alimenticios se deben al inadecuado afrontamiento a los cambios corporales puberales (Smolak, Levine \& Gralen, 1993). Antes, o al inicio de la pubertad, los estudiantes deben recibir información no sólo sobre la menstruación, los óvulos y el esperma humano, los anticonceptivos, el embarazo y el parto, sino también sobre los cambios puberales en la forma corporal, para que los niños y las niñas afronten mejor el proceso de maduración a ser hombre 
o mujer. Es mucho más fácil elaborar estrategias de afrontamiento para la pubertad si los obstáculos son conocidos de antemano, si hay bastante tiempo para prepararse antes de los cambios puberales, y si a uno se le informa sobre los mismos. Para favorecer la validez ecológica de esta investigación, las unidades curriculares de Mi cuerpo y yo fueron enseñadas por los maestros tutores, y no por expertos externos.

\section{Objetivos e hipótesis}

La meta de esta intervención era ayudar al niño y a la niña a sentirse bien con su cuerpo. Se esperaba que los participantes de las clases experimentales, en comparación con las clases de control, manifestarían un mayor incremento de «Conocimiento sobre el desarrollo puberal» y una disminución de «Insatisfacción corporal», «Preocupaciones por la forma corporal»e «Interiorización del ideal delgado».

\section{Method}

\section{Participantes}

Un total de 972 alumnos participaron en este estudio (485 niñas y 484 niños; tres alumnos no contestaron este ítem). Todos ellos cursaban $4^{\circ}$ ó $5^{\circ}$ de educación primaria, y formaban parte de 40 grupos de clase escolares de Alemania (Baden-Wuerttemberg, Saarland) o 2 grupos de clase de Francia (clases de bilingüismo francés-alemán en Nancy). La edad media de la muestra era 10,5 años $(D T=1.1)$. De estas clases, 24 pertenecían a escuelas rurales y 18 a escuelas urbanas o suburbanas. Se asignaron 25 clases $(\mathrm{N}=591 ; 308$ niñas, 282 niños $)$ al grupo de la intervención, y 17 clases $(\mathrm{N}=381 ; 177$ niñas, 202 niños) al grupo control. No existía diferencia significativa entre el grupo de control y el grupo de la intervención en cuanto a edad $\left[t_{(925)}=0.94, p=0.35\right]$, sexo $\left[\chi_{(1)}^{2}=2.79, p=0.10\right]$, o zona rural/urbana $\left[\chi_{(1)}^{2}=0.63\right.$, $p=0.43]$. De estos alumnos, el 97,5\% estuvieron presentes en el momento del pretest, y el $95,7 \%$ en el momento del postest. Sólo los alumnos que hicieron tanto el pretest como el postest (93,2\% de la muestra; 454 niños y 452 niñas) fueron incluidos en el análisis de la eficacia del programa. En el grupo experimental, el 6,1\% de los alumnos estuvieron ausentes en el momento del pre- o postest, y en el grupo control, faltaron un 7,9\% de los alumnos en una de estas mediciones; esta diferencia no es significativa $\left(\left[\chi_{(1)}^{2}=1.16, p=0.28\right]\right.$. Los alumnos que faltaron al pre- o postest no estuvieron disponibles debido a enfermedad, a actividades de la administración escolar, o a cambio de domicilio. 


\section{Intervención}

Con vistas a una futura diseminación más amplia, el programa fue desarrollado como una intervención breve (seis lecciones escolares, es decir, 6 × 45 min) para ser integrada en el horario escolar normal de los estudiantes. Los principales temas y objetivos de las lecciones se presentan en la Tabla 1. Los ejercicios de meditación guiada y de relajación eran elementos céntricos de la intervención, y fueron seleccionados para promover la satisfacción corporal. Los textos sobre la pubertad se formularon para procurar el reconocimiento de que el incremento de grasa en las nalgas, caderas, muslos, brazos superiores, pechos y zona púbica de las chicas es una parte normal y necesaria del crecimiento puberal. Además, los alumnos deben darse cuenta de la enorme variación interindividual en el ritmo del desarrollo puberal. Como parte de la alfabetización mediática de los alumnos, se mostraron películas y fotografías de personas famosas (imágenes reales y modificadas digitalmente) para ejemplificar las distintas formas de manipulación de los medios. Se buscaba hacer entender al alumnado que si desean parecerse a las imágenes mediáticas delgadas y/o musculosas, se adhieren a una imagen corporal y estética inalcanzable.

Tabla 1: Esquema de las unidades curriculares de «Mi Cuerpo y yo»

\begin{tabular}{|c|c|c|c|}
\hline Unidad & Temas principales & Objet & vos \\
\hline 0 & $\begin{array}{l}\text { Tarea de casa: completar un perfil sobre } \\
\text { uno mismo (p.ej. «Cosas que me gustan } \\
\text { de mí mismo») }\end{array}$ & $\circ$ & auto-conciencia \\
\hline \multirow[t]{2}{*}{1} & $\begin{array}{l}\text { Presentación de los perfiles de los alum- } \\
\text { nos durante la clase }\end{array}$ & $\circ$ & autoestima \\
\hline & $\begin{array}{l}\text { Relajación dirigida hacia la apreciación } \\
\text { del cuerpo }\end{array}$ & $\circ$ & $\begin{array}{l}\text { conciencia plena (mindful- } \\
\text { ness) }\end{array}$ \\
\hline \multirow[t]{3}{*}{2} & $1^{\mathrm{a}}$ conferencia: Hacerse mayor & $\circ$ & $\begin{array}{l}\text { comprender y aceptar los } \\
\text { cambios puberales }\end{array}$ \\
\hline & $\begin{array}{l}\text { Ejercicio para formación de equipo: Expe- } \\
\text { riencia del cuerpo }\end{array}$ & $\circ$ & $\begin{array}{l}\text { percibir la experiencia orgá- } \\
\text { nica }\end{array}$ \\
\hline & Meditación dirigida: Me gusto como soy & $\circ$ & aceptar el cuerpo propio \\
\hline
\end{tabular}




\begin{tabular}{|c|c|c|c|}
\hline $3 / 4$ & $\begin{array}{l}2^{\text {a }} \text { conferencia: Cambios en las proporcio- } \\
\text { nes corporales durante la pubertad } \\
\text { (p.ej. deposiciones de grasa normales; } \\
\text { asincronías inter- e intra-individuales. }\end{array}$ & $\circ$ & $\begin{array}{l}\text { considerar normal las caderas } \\
\text { más anchas y el incremento } \\
\text { de grasa durante la pubertad } \\
\text { considerar normal que haya } \\
\text { variedad en el ritmo de desa- } \\
\text { rrollo }\end{array}$ \\
\hline 5 & $\begin{array}{l}\text { Alfabetización mediática (cómo se hace } \\
\text { una chica o chico de portada: iluminación, } \\
\text { manipulación digital, y delgadez / muscu- } \\
\text { losidad) }\end{array}$ & $\circ$ & $\begin{array}{l}\text { desarrollar resistencia hacia } \\
\text { los mensajes culturales de } \\
\text { belleza }\end{array}$ \\
\hline 6 & $\begin{array}{l}\text { Ejercicio para formación de equipo: «Du- } \\
\text { cha caliente» }\end{array}$ & $\circ$ & auto-aceptación \\
\hline & Prueba & & \\
\hline
\end{tabular}

\section{Instrumentos}

La insatisfacción corporal se midió con la versión alemana (Paul \& Thiel, 2005) de la subescala Insatisfacción Corporal del Eating Disorders Inventory (EDI-2) [Cuestionario sobre trastornos alimenticios] (Garner, 1991). La subescala consta de 9 ítems que pretenden evaluar las creencias del individuo sobre la forma y tamaño de partes específicas del cuerpo - las asociadas con incrementos de grasa durante la pubertad - como por ejemplo, «Creo que mis muslos están muy gordos». En la muestra de este estudio, la consistencia interna fue alta, tanto en el pretest (Alfa de Cronbach $=0,86$ ) como en el postest (Alfa de Cronbach $=0,89$ ).

Preocupación por la Forma Corporal se midió con una adaptación infantil de la subescala Shape Concern [Preocupación por la Forma] de la EDE-Q (Fairburn y Beglin, 1994). La subescala Shape Concern mide la insatisfacción y la preocupación por la forma corporal, el miedo a engordar, la importancia de la forma corporal para la persona, los sentimientos de estar gordo, y sentirse incómodo con el cuerpo de uno. Wade, Byrne, y Bryant-Waugh (2008) encontraron buena fiabilidad interna para esta subescala. Tomando en cuenta la versión alemana de la EDE-Q de Hilbert y Tuschen-Caffier (2006), y la adecuación del contenido a la edad, se acortó la longitud de los ítems de la subescala Shape Concern. Para esta escala revisada, se consiguió un coeficiente alfa de Cronbach de 0,83 en el pretest, y 0,86 en el postest. 
La forma corporal femenina ideal se midió con las Body Image Silhouettes (BIS) [siluetas de la imagen corporal] del Kids' Eating Disorder Survey [Cuestionario infantil sobre trastornos alimenticios] (Childress, Jarrell y Brewerton, 1993). En este trabajo, las Body Image Silhouettes (BIS) se usaron como medida de la forma ideal del cuerpo femenino. A los encuestados (niñas y niños) se les pidió que marcasen la figura femenina más guapa (a partir de nueve dibujos de niñas preadolescentes que variaban desde muy delgada hasta obesa). Cada silhueta estaba numerada ( $1=$ la más pequeña; $9=$ la más grande) y por debajo de las siluetas se veía una escala de medida $(1.0,1.1 \ldots 8.9,9.0)$, la que permitió a los encuestados elegir una posición entre dos siluetas si querían. Candy y Fee (1998) evaluaron las propiedades psicométricas de las BIS y encontraron una fiabilidad test-retest después de dos semanas de 0,74 para forma corporal ideal. En este trabajo, la fiabilidad test-retest después de cuatro semanas, para forma corporal femenino ideal, fue 0,71 (para el grupo de control).

Se midió el conocimiento sobre el desarrollo puberal con una escala de 14 ítems, construida por los autores. Esta escala consta únicamente de preguntas tipo verdadero o falso, y mide el conocimiento de los alumnos sobre el desarrollo puberal (ejemplo: «A causa de la pubertad, las niñas acumulan grasa corporal»). El alfa de Cronbach era baja en el pretest ( $\alpha=$ $0,59)$ y buena en el postest $(\alpha=0,74)$.

La Satisfacción con el programa se midió en el postest. Los alumnos del grupo experimental calificaron el programa en conjunto desde 1 (excelente) a 5 (pobre): «¿En general, cómo te parecieron las lecciones de Mi cuerpo y yo?»

\section{Procedimiento}

Este trabajo empleó un diseño de medidas repetidas, comparando las puntuaciones pre- y pos-intervención, en insatisfacción corporal, preocupación por la imagen corporal, forma corporal femenino ideal, y conocimientos sobre el desarrollo puberal. Las clases se asignaron aleatoriamente al grupo experimental o al grupo control. La investigación se llevó a cabo de acuerdo con la Declaración de Helsinki sobre principios éticos para la investigación médica que incluye sujetos humanos. Para un análisis detallado, puede ser importante saber las medidas de índice de masa corporal (IMC) de los participantes. Sin embargo, usar una báscula digital en el aula para saber el peso de los alumnos de primaria puede resultar perjudicial al desencadenar o reforzar comportamientos no saludables de control de peso. Por tanto, nos 
pareció poco ético medir el IMC de los participantes. Los padres, alumnos, y directores de escuela dieron su consentimiento informado después de la presentación de los objetivos y el contenido de Mi cuerpo y yo. La tasa media de respuesta a los cuestionarios de evaluación fue el 99,9\%. En el grupo experimental, los maestros tutores o profesores de biología aplicaron el programa dentro de un periodo de una o dos semanas. Cada profesor recibió materiales didácticos e instrucciones por escrito.

\section{Análisis de datos}

Se utilizó el paquete estadístico SPSS para Windows para las estadísticas descriptivas, comparaciones de grupo, y pruebas de MANOVA y ANOVA. La $d$ de Cohen se utilizó para calcular los tamaños del efecto. El nivel de significación se estableció en $p<0,05$, con una corrección Bonferroni-Holm para comparaciones múltiples.

\section{Resultados}

\section{Satisfacción con el programa}

La satisfacción de los alumnos con el programa fue bastante alta. De los alumnos participantes, el 35\% calificó las lecciones como excelentes, el 39\% como buenas, el 22\% como aceptables, el $2 \%$ como regulares, y el $3 \%$ como no buenas. No hubo diferencia significativa por sexos en el nivel de satisfacción con el programa $\left[t_{(556)}=0,55, p=0,59\right]$.

\section{Eficacia del programa}

Se llevó a cabo un análisis de varianza multivariable (MANOVA), usando un modelo lineal general (MLG), para analizar los efectos del grupo (experimental vs. control) y de sexo (niñas vs. niños) sobre las variables dependientes «Insatisfacción corporal», «Preocupación por la Forma Corporal», «Forma corporal femenina ideal», y «Conocimiento sobre el desarrollo puberal». Los resultados del MANOVA revelaron un efecto principal multivariable significativo de momento $\left[F_{(4,865)}=74,73, p<0,001\right]$, una interacción significativa momento por grupo (experimental vs. control), y un efecto principal significativo de sexo $\left[F_{(4,865)}=29,28\right.$, $p<0,001]$. No hubo interacciones estadísticamente significativas de momento por sexo $[F$ $(4,865)=2,00, p=0,09]$ o de momento por grupo por sexo $\left[F_{(4,865)}=0,34, p=0,85\right]$. Por lo tanto, no hubo necesidad de análisis adicionales separados por sexo. Se llevaron a cabo pruebas de ANOVA para las variables dependientes "Insatisfacción corporal (EDI 2)", "Preocupación por la forma corporal (EDE-Q), "Forma corporal deseada (KEDS)", y "conocimientos 
sobre el desarrollo puberal" con la muestra total. Para compensar la posibilidad de errores de tipo I, debido a múltiples pruebas, se adoptó el nivel convencional de alfa ,05 a través de una corrección Bonferroni-Holm. En la Tabla 2 se pueden observar las puntuaciones medias pretest y postest de los grupos experimental y control, y las pruebas ANOVA de la interacción momento por grupo (efectos de la intervención) para todas las variables dependientes.

Tabla 2. Pruebas de ANOVA para las variables dependientes "Insatisfacción corporal (EDI 2)", “Preocupación por la forma corporal (EDE-Q), “Forma corporal deseada (KEDS)”, y "Conocimientos sobre el desarrollo puberal"

\begin{tabular}{|c|c|c|c|c|c|c|c|}
\hline & \multirow[t]{2}{*}{ Grupo } & \multirow{2}{*}{$\begin{array}{l}\text { Pretest } \\
\qquad M(D T)\end{array}$} & \multirow{2}{*}{$\begin{array}{l}\text { Postest } \\
\qquad M(D T)\end{array}$} & \multicolumn{4}{|c|}{ Momento por grupo } \\
\hline & & & & $\boldsymbol{F}$ & $p$ & $\eta^{2}$ & $g l$ \\
\hline \multirow{2}{*}{$\begin{array}{l}\text { Insatisfacción } \\
\text { corporal (EDI 2) }\end{array}$} & GE & $3,22(, 67)$ & $3,26(, 69)$ & \multirow{2}{*}{ 7,97 } & \multirow{2}{*}{, $005^{*}$} & \multirow{2}{*}{01} & \multirow{2}{*}{$1 / 870$} \\
\hline & $\mathrm{GC}$ & $3,26(, 61)$ & $3,23(, 70)$ & & & & \\
\hline \multirow{2}{*}{$\begin{array}{l}\text { Preocupación por } \\
\text { la forma corporal } \\
(\text { EDE-Q) }\end{array}$} & GE & $3,04(, 74)$ & $3,08(, 77)$ & \multirow[b]{2}{*}{6,73} & \multirow[b]{2}{*}{, $010^{*}$} & \multirow[b]{2}{*}{01} & \multirow[b]{2}{*}{$1 / 870$} \\
\hline & $\mathrm{GC}$ & $3,17(, 76)$ & $3,12(, 80)$ & & & & \\
\hline \multirow{2}{*}{$\begin{array}{l}\text { Forma corporal } \\
\text { deseada }(\text { KEDS) }\end{array}$} & GE & $3,33(, 80)$ & $3,45(, 71)$ & \multirow{2}{*}{5,51} & \multirow{2}{*}{, $019^{*}$} & \multirow{2}{*}{,01 } & \multirow{2}{*}{$1 / 870$} \\
\hline & GC & $3,23(, 77)$ & $3,24(, 78)$ & & & & \\
\hline \multirow{3}{*}{$\begin{array}{l}\text { Conocimientos } \\
\text { sobre el desarrollo } \\
\text { puberal }^{\mathrm{d}}\end{array}$} & GE & $9,51(1,78)$ & $11,46(1,83)$ & \multirow{3}{*}{226,9} & \multirow{3}{*}{, $000^{*}$} & \multirow{3}{*}{,21 } & \multirow{3}{*}{$1 / 870$} \\
\hline & & & & & & & \\
\hline & $\mathrm{GC}$ & $9,26(2,04)$ & $9,39(2,10)$ & & & & \\
\hline
\end{tabular}

Notas: ${ }^{a}$ Puntuaciones más altas indican mayor satisfacción con el cuerpo de uno $(\min .=1, \max .=4)$.

${ }^{\mathrm{b}}$ Puntuaciones más altas indican menos preocupación por la forma corporal $(\min .=1, \max .=4)$.

${ }^{\mathrm{b}}$ Puntuaciones más altas indican menos interiorización del ideal delgado $(\min .=1, \max .=9)$.

${ }^{\mathrm{d}}$ Puntuaciones más altas indican mayor conocimiento sobre el desarrollo puberal $(\min .=0, \max .=14)$.

$\mathrm{GE}=$ Grupo Experimental, $\mathrm{CG}=$ Grupo de Control

$*=$ valores de $\mathrm{p}$ significativos después de las correcciones post-hoc Bonferroni-Holm debido a pruebas múltiples

Desarrollo puberal

Los efectos de interacción significativos de momento (pretest vs. postest) por grupo (experimental vs. control) se observaron para todas las variables dependientes. En comparación con el grupo de control, los alumnos del grupo experimental mostraron un incremento significativo de satisfacción corporal, una disminución de preocupación por la forma corporal, un mayor alejamiento del ideal del cuerpo femenino delgado y un mayor incremento de conocimiento sobre el desarrollo puberal. Los tamaños del efecto de los impactos del programa se 
calcularon según la $d$ de Cohen como la diferencia entre las puntuaciones pretest y postest, dividido por la desviación típica combinada. Para el incremento de satisfacción corporal, se calculó un tamaño de efecto de $d=0,12$; para la disminución de preocupación por la forma corporal, $d=0.11$; para el alejamiento del ideal del cuerpo delgado, $d=0.13$; y para el incremento de conocimientos sobre el desarrollo puberal, $d=0.89$.

Un análisis de datos post hoc del efecto principal de sexo reveló que las niñas, en comparación con los niños, estaban más insatisfechas con sus cuerpos, tenían más preocupación por su forma corporal, y eligieron las figuras femeninas más delgadas como el ideal. Las puntuaciones de las niñas en "Conocimientos sobre el desarrollo puberal" eran más altas que las puntuaciones de los niños. Curiosamente, hubo una excepción llamativa: En comparación con los niños, las niñas sabían menos sobre la acumulación de grasa durante la pubertad $\left[t_{(739)}\right.$ $=5,22, p<0,001]$. En el pretest, sólo el $17 \%$ de las niñas del grupo experimental sabían que la acumulación de grasa es una parte normal del proceso puberal, mientras en el postest, el $76 \%$ de las niñas sabían la respuesta correcta $\left[\chi_{(1)}^{2}=162,66, p<0,001\right]$. Además, en el pretest, sólo el 35\% de las niñas del grupo experimental sabían que las caderas se ensanchan durante la pubertad para facilitar el parto. En el postest, el 90\% de las niñas del grupo experimental sabían la respuesta correcta $\left[\chi_{(1)}^{2}=145,29, p<0,001\right]$, mientras que no hubo cambio significativo en el grupo control.

\section{Discusión}

El propósito de esta investigación ha sido probar la eficacia del programa escolar modificado Mi Cuerpo y yo, el cual fue desarrollado para mejorar la satisfacción corporal en niñas y niños preadolescentes. Se esperaba que la participación en las lecciones de Mi Cuerpo y yo favorecería los conocimientos de los alumnos sobre el desarrollo puberal y disminuiría la insatisfacción corporal, la preocupación por la imagen corporal y la interiorización del ideal delgado. Hasta la fecha, sólo se pudieron encontrar cuatro estudios bien diseñados sobre intervenciones escolares con grupo control (Bird et al., 2013; Kater et al., 2000; Kater et al., 2002; Smolak et al., 1998a, 1998b; Stock et al., 2007). Los hallazgos de los cuatro estudios comprobaron la eficacia de los programas desarrollados, pero no llegaron a evidenciar un impacto sobre la insatisfacción corporal en niñas y niños también. Los hallazgos de esta investigación indican que el programa Mi Cuerpo y yo fue eficaz no sólo para incrementar los conocimientos sobre el desarrollo puberal, sin también para disminuir la insatisfacción corporal, la preocupación por la imagen corporal y la interiorización del ideal delgado. 
El programa desarrollado no era relevante únicamente para las niñas. Es notable que la interacción momento por grupo por sexo no era significativa, y que la satisfacción con el programa de los niños no era menos que la de las niñas. Especialmente destacable es el nivel de ignorancia de las niñas sobre la acumulación de grasa, en el momento del pretest, y su mayor conocimiento en el postest (este resultado es acorde con los hallazgos de Smolak, Levine y Schermer, 1998a). Los datos de esta investigación aportan evidencia de que Mi cuerpo y yo consigue concienciar tanto a niñas como a niños de la enorme variedad de los cambios puberales entre individuos. Los niños y niñas aprendieron que la edad del inicio de la pubertad varía entre individuos y que todos tienen su propio ritmo biológico. Mientras que la investigación previa ha aportado evidencias de que el conocimiento de los trastornos alimenticios y sus características (el vómito autoinducido, el abuso de laxantes, y la inanición) puede llevar a que los alumnos imiten estos métodos nada saludables para controlar su peso (Carter, Stewart, Dunn, y Fairburn, 1997; O’Dea y Abraham, 2000), los resultados de esta investigación sugieren que el conocimiento sobre el desarrollo puberal puede tener un impacto beneficioso en la satisfacción corporal.

Aunque resulten alentadores los efectos momento por grupo, logrados aquí, es necesario reconocer varias limitaciones de esta investigación. En primer lugar, los efectos a corto plazo no son una predicción de resultados a largo plazo. Basándose en esta investigación, no es posible establecer si los beneficios de la intervención disminuirán, desaparecerán, o se mandentrán a largo plazo. Segundo, este trabajo ha dependido de auto-informes, lo cual puede reducir la fiabilidad de los datos. Tercero, según los criterios de Cohen, todos los efectos de la intervención en la satisfacción corporal han sido bastante más pequeños que la diferencia mínima clínicamente importante (DMCI) para ensayos clínicos; sólo el efecto en conocimiento era grande. Sin embargo, estos hallazgos son acordes con los resultados del meta-análisis realizado por Stice y Shaw (2004). Además, los efectos de techo podían contribuir a los efectos pequeños de la intervención porque en el momento del pretest, las puntuaciones medias de satisfacción corporal eran relativamente altas, limitando la capacidad de mejora.

A pesar de ello, el trabajo actual tiene algunos puntos fuertes metodologícos específicos. En primer lugar, el tamaño de la muestra es relativamente grande; segundo, hay un grupo de control adecuado; tercero, las medidas son adaptadas para niños y su fiabilidad es buena o aceptable; y finalmente, la corta duración del programa preventivo y la implementación por maestros de escuela en lugar de desarrolladores del programa u otros distribuidores profesio- 
nales incrementa la validez ecológica de esta investigación. El programa Mi cuerpo y yo no tiene requisitos previos ni requiere habilidades especiales, y por tanto es fácil de implementar. Stice, Shaw, y Marti (2007) habían encontrado que eran más eficaces los programas de prevención de trastornos alimenticios aplicados por expertos externos que los aplicados por los maestros. Por consiguiente, en el contexto de esta revisión meta-analítica, los resultados de la investigación actual son alentadores.

Un desafío para el futuro es examinar los efectos del programa a largo plazo. Esto requiere el desarrollo e implementación de repetidas sesiones de refuerzo y de medidas continuadas. Por otra parte, en el grupo experimental, puede que las técnicas de investigación cualitativa ofrezcan una mejor comprensión de las experiencias didácticas y las interacciones dentro del aula.

Los programas escolares de prevención deben comenzar en la escuela primaria, porque es difícil cambiar actitudes negativas muy arraigadas hacia el cuerpo y la forma de uno mismo. Son necesarios enfoques coeducativos y de corta duración para facilitar la implementación en un contexto escolar atareado y muchas veces estresante. Los efectos del programa en un ambiente coeducativo natural fueron alentadores en el corto plazo, sobre todo en el contexto de los resultados de la investigación previa. En resumen, el trabajo actual indica que $M i$ Cuerpo y yo tiene el potencial de ser un programa escolar existoso a la hora de promover la satisfacción corporal en niñas y niños preadolescentes, antes de que lleguen a prevalecer la preocupación no saludable por la forma corporal y los trastornos alimenticios.

\section{Reconocimientos}

La elaboración de este artículo fue subvencionado con un subsidio de la Universidad de la Educación de Ludwigsburg.

\section{Referencias}

Becker, A. E. (2004). Television, disordered eating, and young women in Fiji: negotiating body image and identity during rapid social change. Culture, Medicine and Psychiatry, 28, 533-559. http://dx.doi.org/10.1007/s11013-004-1067-5

Bennaroch, A., Pérez, S., y Perales, J. (2011). Factors influencing adolescent eating behavior: Application and validation of a diagnostic instrument. Electronic Journal of Research in Educational Psychology, 9(3), 1219-1244. 
Bird, E. L., Halliwell, E., Diedrichs, P. C., y Harcourt, D. (2013). Happy Being Me in the UK: a controlled evaluation of a school-based body image intervention with pre-adolescent children. Body Image, 10, 326-334. http://dx.doi.org/10.1016/j.bodyim.2013.02.008

Bird, P. J. (2006). Why does fat deposit on the hips and thighs of women and around the stomachs of men? Scientific American, May 15, 125-127. Recuperado de: http://www.scientificamerican.com/article.cfm?id=why-does-fat-deposit-on-t

Birkeland, R., Thompson, J. K., Herbozo, S., Roehrig, M., Cafri, G., y van den Berg, P. (2005). Media exposure, mood, and body image dissatisfaction: an experimental test of person versus product priming. Body Image, 2, 53-61. http://dx.doi.org/10.1016/j.bodyim.2004.11.002

Candy, C. M., y Fee, V. E. (1998). Reliability and concurrent validity of the Kids' Eating Disorders Survey (KEDS) Body Image Silhouettes with preadolescent girls. Eating Disorders, 6, 297-308.

Carter, J. C., Stewart, D. A., Dunn, V. J., y Fairburn, C. G. (1997). Primary Prevention of Eating Disorders: Might it do more harm than good? International Journal of Eating Disorders, 22, 167-172.

Childress, A. C., Jarrell, M. P., \& Brewerton, T. D. (1993). The Kids' Eating Disorders Survey (KEDS): Internal consistency, component analysis, and reliability. Eating Disorders 1, 123-133.

Cohen, S. B. (2006). Media exposure and the subsequent effects on body dissatisfaction, disordered eating, and drive for thinness: a review of the current research. Mind Matters: The Wesleyan Journal of Psychology, 1, 57-71. Recuperado de: http://www.ddwei.info/pdf/subsequent/4.pdf

Dohnt, H. K., y Tiggemann, M. (2008). Promoting positive body image in young girls: an evaluation of ,Shapesville'. European Eating Disorders Review, 16, 222-233. http://dx.doi.org/10.1002/erv.814

Fairburn, C. G., y Beglin, S. J. (1994). Assessment of eating disorders: interview or selfreport questionnaire? International Journal of Eating Disorders, 16, 363-370.

Garner, D. M. (1991). Eating Disorder Inventory - 2 Professional Manual. Odessa, FL: Psychological Assessment Resources Inc.

Greenglass, E. R. (2002). Proactive coping. En E. Frydenberg (Ed.), Beyond coping: Meeting goals, vision, and challenges (págs. 37-62). Londres: Oxford University Press.

Hilbert, A., y Tuschen-Caffier, B. (2006). Eating Disorder Examination - Questionnaire Deutschsprachige Übersetzung [traducción alemana]. Münster: Verlag für Psychotherapie.

Hinz, A., \& Denner, C. (2007). Prävention essstörungsrelevanter Körperbildwahrnehmungen: Entwicklung und Evaluation einer Unterrichtseinheit in Klasse 4. Zeitschrift für Empirische Pädagogik, 21, 271-290.

Holt, K. E., y Ricciardelli, L. A. (2008). Weight concerns among elementary school children: a review of prevention programs. Body Image, 5, 233-243. http://dx.doi.org/10.1016/j.bodyim.2008.02.002.

Kater, K. J., Rohwer, J., y Levine, M. P. (2000). An elementary school project for developing healthy body image and reducing risk factors for unhealthy and disordered eating. Eating Disorders, 8, 3-16. 
Kater, K. J., Rohwer, J., y Londre, K. (2002). Evaluation of an upper elementary school program to prevent body image, eating and weight concerns. Journal of School Health, 72, 199-204. http://dx.doi.org/10.1111/j.1746-1561.2002.tb06546.x

Levine, M. P., y Smolak, L. (2001). Primary prevention of body image disturbances and disordered eating in childhood and early adolescence. En J. K. Thompson y L. Smolak (Eds.), Body image, eating disorders, and obesity in youth: assessment, prevention, \& treatment (pp. 237-260). Washington, DC: American Psychological Association.

Levine, M. P., y Smolak, L. (2007). Prevention of negative body image, disordered eating, and eating disorders: an update. En S. Wonderlich, J. Mitchell, M. De Zwaan \& H. Steiger (Eds.), Annual review of eating disorders: Part I -- 2007 (pp. 1-14). Oxford: Radcliffe.

López-Guimerà, G., Levine, M. P., Sánchez-Carracedo, D., y Fauquet, J. (2010). Influence of mass media on body image and eating disordered attitudes and behaviors in females: a review of effects and processes. Media Psychology, 13, 387-416. http://dx.doi.org/10.1080/15213269.2010.525737

Lunde, C. (2009). What people tell you gets to you. Body satisfaction and peer victimization in early adolescence. Tesis doctoral, Departamento de Psicología, Universidad de Gothenburg, Suecia. Recuperado de: https://gupea.ub.gu.se/bitstream/2077/19350/1/gupea_2077_19350_1.pdf

Malina, R. M., y Bouchard, C. (1991). Growth, maturation, and physical activity. Illinois: Human Kinetics Books.

Markey, C. N., y Markey, P. M. (2011). Body Image. En R. J. R. Levesque (Ed.), Encyclopedia of Adolescence (pp. 310-320). Nueva York: Springer.

Mond, J., van den Berg, P., Boutelle, K., Hannan, P., y Neumark-Sztainer, D. (2011). Obesity, body dissatisfaction, and emotional well-being in early and late adolescence: Findings from the project EAT study. Journal of Adolescent Health, 48, 373-378. http://dx.doi.org/10.1016/j.jadohealth.2010.07.022

O'Dea, J. A. (2005). School-based health education strategies for the improvement of body image and prevention of eating problems. An overview of safe and successful interventions. Health Education, 105, 11-33. Recuperado de: http://citeseerx.ist.psu.edu/viewdoc/download?doi=10.1.1.485.727\&rep=rep1\&type=p df

O'Dea, J. A., y Abraham, S. (2000). Improving the body image, eating attitudes, and behaviours of young male and female adolescents: a new educational approach that focuses on self-esteem. International Journal of Eating Disorders, 28, 43-57. http://dx.doi.org/10.1002/(SICI)1098-108X(200007)28:13.0.CO;2-D

Paul, T., y Thiel, A. (2005). EDI-2 (Eating Disorder Inventory-2). Versión alemana. Göttingen: Hogrefe.

Piran, N. (2005). Prevention of eating disorders: a review of outcome evaluation research. Israel Journal of Psychiatry and Related Sciences, 42, 172-177. Recuperado de: http://doctorsonly.co.il/wp-content/uploads/2011/12/2005_3_5.pdf

Richardson, S. M., y Paxton, S. J. (2010). An evaluation of a body image intervention based on risk factors for body dissatisfaction: a controlled study with adolescent girls. International Journal of Eating Disorders, 43, 112-122. http://dx.doi.org/10.1002/eat.20682 
Schwarzer, R., y Taubert, S. (2002). Tenacious goal pursuits and striving toward personal growth: Proactive coping. En E. Frydenberg (Ed.), Beyond Coping: Meeting Goals, Visions, and Challenges (págs. 20-35). Londres: Oxford University Press.

Sepúlveda, A. R., y Calado, M. (2012). Westernization: the role of mass media on body image and eating disorders. En I. Jáuregui-Lobera (Ed.), Relevant topics in eating disorders (págs. 47-64). InTech. http://dx.doi.org/10.5772/31307

Smolak, L., Levine, M. P., y Gralen, S. (1993). The impact of puberty and dating on eating problems among middle school girls. Journal of Youth and Adolescence, 22, 355-368.

Smolak, L., Levine, M. P., y Schermer, F. (1998a). A controlled evaluation of an elementary school primary prevention program for eating problems. Journal of Psychosomatic Research, 44, 339-353.

Smolak, L., Levine, M. P., y Schermer, F. (1998b). Lessons from lessons: an evaluation of an elementary school prevention program. En W. Vandereycken \& G. Noordenbos (Eds.), The prevention of eating disorders (pp. 137-172). Nueva York: New York University Press.

Steinberg, L. (2010). Adolescence (9a ed.). Nueva York: McGraw-Hill.

Steinhausen, H.-C. (2002). The outcome of anorexia nervosa in the $20^{\text {th }}$ century. American Journal of Psychiatry, 159, 1284-1293. http://dx.doi.org/10.1176/appi.ajp.159.8.1284

Stice, E. (2002). Risk and maintenance factors for eating pathology: A meta-analytic review. Psychological Bulletin, 128, 825-848. http://dx.doi.org/10.1037//0033-2909.128.5.825

Stice, E., y Shaw, H. (2004). Eating disorder prevention programs: a meta-analytic review. Psychological Bulletin, 130, 206-227. http://dx.doi.org/10.1037/0033-2909.130.2.206

Stice, E., Shaw, H., y Marti, N. (2007). A meta-analytic review of eating disorder prevention programs: encouraging findings. Annual Review of Clinical Psychology, 3, 207-231. http://dx.doi.org/10.1146/annurev.clinpsy.3.022806.091447

Stock, S., Miranda, C., Evans, S., Plessis, S., Ridley, J., Yeh, S., y Chanoine, J.-P. (2007). Healthy Buddies: a novel, peer-led health promotion program for the prevention of obesity and eating disorders in children in elementary school. Pediatrics, 120, 10591068. http://dx.doi.org/10.1542/peds.2006-3003

Susman, E. J., y Rogol, A. (2004). Puberty and psychological development. En R. M. Lerner y L. Steinberg (Eds.), Handbook of adolescent psychology (2 ${ }^{\mathrm{a}}$ ed.) (págs. 15-44). New Jersey: John Wiley \& Sons.

Wade, T. D., Byrne, S., y Bryant-Waugh, R. (2008). The eating disorder examination: norms and construct validity with young and middle adolescent girls. International Journal of Eating Disorders, 41, 551-558. http://dx.doi.org/10.1002/eat.20526

Walker, K. (2007). Mechanisms of self-esteem change in overweight children participating in a family-based weight management program. Tesis doctoral, Universidad de Florida. Recuperado de: http://ufdcimages.uflib.ufl.edu/UF/E0/02/11/27/00001/walker_k.pdf 